\title{
Elevated type I interferon-like activity in a subset of multiple sclerosis patients: molecular basis and clinical relevance
}

\author{
Alexander Hundeshagen ${ }^{1 \dagger}$, Michael Hecker ${ }^{1,2^{*}+}$, Brigitte Katrin Paap ${ }^{1,2}$, Charlotte Angerstein ${ }^{1}$, Ole Kandulski ${ }^{1}$, \\ Christian Fatum', Christiane Hartmann ${ }^{1}$, Dirk Koczan², Hans-Juergen Thiesen ${ }^{2}$ and Uwe Klaus Zettl ${ }^{1}$
}

\begin{abstract}
Background: A subset of patients with multiple sclerosis (MS) shows an increased endogenous IFN-like activity before initiation of IFN-beta treatment. The molecular basis of this phenomenon and its relevance to predict individual therapy outcomes are not yet fully understood. We studied the expression patterns of these patients, the prognostic value of an elevated IFN-like activity, and the gene regulatory effects of exogenously administered IFN-beta.

Methods: Microarray gene expression profiling was performed for 61 MS patients using peripheral blood mononuclear cells obtained before and after 1 month of IFN-beta therapy. Expression levels of genes involved in pathways either inducing or being activated by IFN-beta were compared between patients with high ( $M X 1_{\text {high }}$ cohort) and low ( $M X 1_{\text {low }}$ cohort) endogenous IFN-like activity. Patients were followed for 5 years and relapses as well as progression on the expanded disability status scale (EDSS) were documented.

Results: Before the start of therapy, 11 patients presented elevated mRNA levels of IFN-stimulated genes indicative of a relatively high endogenous IFN-like activity $\left(M X 1_{\text {high }}\right)$. In these patients, pathogen receptors (for example, TLR7, R/G-I and IFIH1) and transcription factors were also expressed more strongly, which could be attributed to an overactivity of IFN-stimulated gene factor 3 (ISGF3, a complex formed by STAT1, STAT2 and IFN regulatory factor 9). After 1 month of IFN-beta therapy, the expression of many pathway genes was significantly induced in $M X 1_{\text {low }}$ patients, but remained unaltered in $M X 1_{\text {high }}$ patients. During follow-up, relapse rate and changes in EDSS were comparable between both patient groups, with differences seen between different types of IFN-beta drug application.

Conclusions: Therapeutic IFN-beta induces the transcription of several genes involved in IFN-related pathways. In a subgroup of MS patients, the expression of these genes is already increased before therapy initiation, possibly driven by an overexpression of ISGF3. Patients with high and low endogenous IFN-like activity showed similar clinical long-term courses of disease. Different results were obtained for different IFN-beta drug preparations, and this merits further investigation.
\end{abstract}

Keywords: Blood biomarker, IFN-beta, Multiple sclerosis, MX1, Pharmacological effects, Signaling pathway, Therapy response

\footnotetext{
* Correspondence: michael.hecker@rocketmail.com

${ }^{\dagger}$ Equal contributors

'Department of Neurology, Division of Neuroimmunology, University of Rostock, Gehlsheimer Str. 20, 18147 Rostock, Germany

${ }^{2}$ Institute of Immunology, University of Rostock, Schillingallee 68, 18057

Rostock, Germany
}

\section{Ciomed Central}

(c) 2012 Hundeshagen et al.; licensee BioMed Central Ltd. This is an Open Access article distributed under the terms of the Creative Commons Attribution License (http://creativecommons.org/licenses/by/2.0), which permits unrestricted use, distribution, and reproduction in any medium, provided the original work is properly cited. 


\section{Background}

Multiple sclerosis (MS) is a chronic inflammatory disease causing neuronal demyelination and axonal decay in the central nervous system. The impaired nerve conduction results in progressive neurological dysfunctions, ultimately leading to severe impairment of the patient [1]. No distinct etiology has so far been determined, but autoimmune processes, genetic dispositions and environmental factors have been associated with the disease [1-5]. The individual course of MS is highly heterogeneous making predictions of long-term prognosis challenging. The majority of patients experience a relapsing-remitting type of MS (RRMS) during the first years of disease with episodically flourishing neurological dysfunctions [6-8]. To date, no curative therapy is available. An immunomodulatory therapy with IFN-beta is a common first-line approach for controlling exacerbations and progression in RR-MS. Three drug formulations of IFN-beta are available: subcutaneous (sc) IFN-beta-1a and IFN-beta-1b, and intramuscular (im) IFN-beta-1a [9,10]. The molecular mechanisms of action of these drugs have been studied extensively over the past years $[11,12]$. IFN-beta therapy, however, proved only partially effective, with a subset of patients not sufficiently responding to therapy. This means that, despite treatment, further disease progression can be observed in terms of relapses, worsening on the expanded disability status scale (EDSS), or formation of new brain lesions [13].

A recent focus of research has been the search for molecular biomarkers that can assist in diagnosis as well as in monitoring of disease activity and therapeutic effects. In this context, biomarkers are sought to predict longterm disease progression and individual therapy response with the aim of reducing the delay of effective therapy and lowering socioeconomic costs on ineffective drug applications $[14,15]$. However, none of the potentially prognostic biomarker candidates have so far been established and implemented in clinical practice. More than a hundred genes have been proposed as blood biomarkers, but most could not be independently confirmed in a recent study [16]. One widely accepted explanation for IFN-beta therapy failure is the formation of neutralizing antibodies (NAb). This occurs after a few months of treatment in $7 \%$ to $35 \%$ of the patients, dependent on the drug formulation [17]. NAbs are antibodies against recombinant IFN-beta that diminish its ability to induce the transcription of interferonstimulated genes (ISGs) and hence reduce the bioactivity and the long-term clinical effects of the drug $[18,19]$. Thus far, hundreds of ISGs have been identified. They show a strong co-regulation upon stimulation with type I IFNs (IFN-alpha and IFN-beta) [20,21]. The mRNA induction of ISGs by IFN-beta therapy (in the absence of NAbs) has been demonstrated to be relatively short- lasting, with many ISGs returning to pre-treatment levels within 4 days $[22,23]$. One of the most prominent ISGs is myxovirus resistance protein 1 ( $M X 1$ or $M x A)$. The blood expression of $M X 1$ has been shown to reflect the immunoregulatory activity of IFN-beta, and hence $M X 1$ has been clinically implemented to measure the effects of IFN-beta administration on gene regulation and to detect the presence of NAbs [24,25].

While $M X 1$ is a useful biomarker during IFN-beta treatment, it has been debated whether already prior to treatment the expression of $M X 1$ or other ISGs allows the prediction of individual long-term clinical outcomes. It has been observed that a subgroup of therapy-naïve MS patients shows an elevated endogenous ISG expression and, thus, an increased type I IFN-like activity [26]. Based on this finding, van Baarsen et al. [27] suggested that the pre-treatment IFN signature in the peripheral blood could serve as a prognostic biomarker of clinical responses to IFN-beta therapy. Comabella et al. [28,29] later reported an overexpression of ISGs before the start of IFN-beta treatment in clinical non-responders compared to responders. They observed that, upon treatment, the expression of these genes remained unaltered in non-responders, but was strongly upregulated in responders. They attributed the elevated IFN signature to increased amounts of phosphorylated STAT1 and IFN-alpha/beta receptor 1 (IFNAR1) in monocytes. Moreover, higher baseline levels of IFN-beta itself were detected in non-responders by Axtell et al. [30] and this was later confirmed by Bustamante et al. [29]. On the other hand, Feng et al. [31] detected a lower expression of ISGs in patients with active MS and found that this was linked to a subnormal phosphorylation of the transcription factor (TF) STAT1. They concluded that a defect in type I IFN signaling may predict the course of disease and responses to therapy. Recently, van der Voort et al. [32] studied a group of 116 RR-MS patients and observed that high $M X 1$ mRNA levels in the blood were significantly associated with a longer time to a first new relapse. A potentially beneficial effect of an elevated endogenous type I IFN response was also reported by Hesse et al. [33], who described a positive correlation of $M X 1$ expression and $I L-10$ expression, and a negative correlation of $I L-10$ expression and disease activity on magnetic resonance imaging in untreated MS patients.

The results of these studies have been somewhat inconsistent, possibly due to differences in the type of specimen analyzed, measurement technology, treatment strategy, definition of disease progression, and data analysis. Hence, there is a need to independently validate whether the individual IFN signaling activity, reflected by the expression of $M X 1$ and other ISGs, can predict the individual course of disease. Moreover, the underlying molecular physiology of type I IFN-like activity and 
the effects of IFN-beta therapy on the IFN-beta-related pathways have so far not been elucidated in a comprehensive manner. A deeper insight into these effects may help to better understand the mechanisms of action of the drug and to disclose transcript-based disease heterogeneity.

In this work, we investigated the molecular basis of high endogenous IFN-like activity by studying the pathways involved in IFN regulation and signaling. Furthermore, we analyzed the gene regulatory effects of IFN-beta therapy and the expression differences between MS patients with low and high pre-treatment IFN-like activity. To evaluate the prognostic power of this activity on therapy success, we examined the disease progression over the long-term course of MS.

\section{Methods}

\section{Interferon pathways}

To unravel the molecular basis that accounts for individual differences in the endogenous IFN-like activity, we looked for genes involved either in the pathways regulating IFN-beta expression or in the pathways triggered by IFN-beta. We searched the PubMed database for review articles published within the last 5 years addressing the respective IFN-beta-related pathways. Eleven reviews were selected [34-44], and we extracted the genes that were redundantly mentioned in these publications together with their mutual interactions (Additional file 1). A network of the genes visualizing the different types of interactions (for example, binding, activation and inhibition) was constructed using the Cytoscape software version 2.8.1 (Cytoscape Consortium, San Diego, CA, USA, http://www.cytoscape.org).

\section{Experimental setup and microarray data}

This study comprises 61 patients suffering from RR-MS diagnosed according to the McDonald criteria [45]. The patients were prescribed sc IFN-beta-1a $(n=12)$, sc IFNbeta-1b $(n=25)$ or im IFN-beta-1a $(n=24)$ treatment. Blood samples were drawn immediately before the start of therapy (baseline) and after 1 month prior to the next drug application. Peripheral blood mononuclear cells (PBMC) were separated from the blood samples by Ficoll gradient and total RNA was extracted using the RNeasy Mini Kit (Qiagen, Hilden, Germany). The RNA was processed, labeled and hybridized to Affymetrix HG-U133 A and B or Plus 2.0 oligonucleotide microarrays according to the manufacturer's protocols. To calculate the gene expression levels, we used custom chip definition files (CDFs) and applied the MAS5.0 algorithm. The custom CDFs (version 2.1.0, http://www.xlab.unimo.it/GA_CDF/) [46] define probesets composed of probes matching only a single gene based on the GeneAnnot (version 1.9) and GeneCards (version 2.41) databases [47]. In this way, an accurate assignment of probesets and genes could be achieved. This gave us an expression data set of about 17,000 genes, corrected for variation between the microarrays by loess normalization. The full data are available in the GEO database [GEO:GSE19285, GEO:GSE24427, GEO:GSE33464]. Further details on the experiments and the data preprocessing can be found elsewhere [48,49]. The studies were approved by the University of Rostock's ethics committee and carried out according to the Declaration of Helsinki. Written informed consent was obtained from all patients before the blood sampling.

\section{Statistical analysis of the gene expression data}

Based on the baseline $M X 1$ transcript levels as a measure of endogenous type I IFN-like activity, the patients were divided into two groups. The detected MX1 signal intensities varied from 875 to 11,588 (Additional file 2). We set a cut-off at 3,000, which was two standard errors above the mean. Patients with $M X 1$ levels below the cutoff were allocated to the $M X 1_{\text {low }}$ cohort and those with MX1 levels above the cut-off were allocated to the $M X 1_{\text {high }}$ cohort. The mean expression levels of the genes involved in the IFN-beta-related pathways were then compared between the $M X 1_{\text {high }}$ group and the $M X 1_{\text {low }}$ group at baseline as well as after 1 month using the Wilcoxon rank-sum test. We further compared the expression levels at both time-points within each group using the Wilcoxon signed-rank test. Expression differences with $P$-values below 0.01 were considered statistically significant. For the gene expression comparisons, only data from patients who received IFN-beta sc were considered since, due to the different drug application schedules for IFN-beta sc (3 to 4 injections per week) and IFN-beta im (1 injection per week), the interval between two subsequent drug injections differs. Since the blood sampling was always performed before the next injection, this has a considerable influence on the measured mRNA levels [22,23]. Statistical analyses have been computed with PASW Statistics version 18.0.0 (SPSS Inc., Chicago, IL, USA) and the data were imported into Cytoscape to visualize differences and changes in gene expression.

\section{Validation of the microarray data by real-time PCR}

Quantitative real-time reverse transcription PCR was performed to confirm the gene expression levels quantified by the microarrays. The microarray experiments were conducted between 2003 and 2007, and the real-time PCR experiments were performed in 2012. Meanwhile, the total RNA samples were stored at $-25{ }^{\circ} \mathrm{C}$. RNA quantity and quality was determined using a NanoDrop 1000 Spectrophotometer (Thermo Scientific, Wilmington, DE, USA) and a 2100 Bioanalyzer (Agilent Technologies, Palo Alto, CA, USA), respectively. For 44 of the 61 patients, 
sufficient amounts of PBMC RNA were available for both the baseline sample and the sample obtained 1 month after the start of treatment. A set of genes was remeasured by real-time PCR in the samples of these patients. From each sample, $0.2 \mu \mathrm{g}$ total RNA was reverse transcribed using the High Capacity cDNA Reverse Transcription Kit (Applied Biosystems, Carlsbad, CA, USA). The obtained cDNA was then measured in 384-well TaqMan Arrays with pre-designed TaqMan assay reagents according to the manufacturer's instructions with a 7900HT Sequence Detection System (Applied Biosystems). The two samples from each patient were always processed in one batch. Fluorescence was measured during each PCR cycle, and thresholds at which the signal is detected above background ( $\mathrm{Ct}$ values) were computed automatically using the SDS 2.3 and RQ Manager 1.2 software (Applied Biosystems). For four patients, the real-time PCR measurement was replicated. For those, we calculated the mean of the $\mathrm{Ct}$ values. The $\mathrm{Ct}$ values were then utilized for the analysis of differential expression applying Wilcoxon tests as described for the microarray data. Spearman's rank correlation coefficients and $P$-values were computed to assess whether the real-time PCR data correlate with the microarray data.

\section{Stability of MX1 mRNA expression}

An elevated type I IFN-like activity may either be characteristic for some patients or may be only a temporary phenomenon at the time of blood sampling (for example, due to an undiagnosed viral infection). We therefore evaluated whether the natural $M X 1$ expression is stable over time using the Affymetrix microarray data by Karlovich et al. [GEO:GSE16028] [50]. The data provide whole blood gene expression levels of 22 healthy individuals measured at five different time-points spanning a period of 6 months. We pre-processed the data in the same manner as we pre-processed our own data (see section "experimental setup and microarray data"). Correlation of $M X 1$ levels at study onset and after 6 months was assessed by Spearman's rho. We performed an analysis of variance (ANOVA) to explore the impact of age, gender, time-point and the individual subjects on the measured MX1 transcript signals. The linear ANOVA model was fitted using the $\mathrm{R}$ package "car" with type II sums of squares and F-test statistic.

\section{Analysis of transcription factor binding sites}

We examined the regulatory regions of all genes involved in the IFN-beta-related signaling pathways for putative DNA-binding sites for the transcription factor complex ISGF3 (IFN-stimulated gene factor 3, Transfac database sequence motif identifier V\$ISRE_01). Predictions of evolutionarily conserved transcription factor binding sites (TFBS) provided by the tfbsConsSites track (hg18) of the UCSC database were used for this purpose. For each gene, the regulatory region was assumed to be 1,000 base pairs up- and downstream of the respective transcription start site, which was taken from the GeneCards database (version 2.41).

\section{Clinical data evaluation}

All patients were monitored for the occurrence of relapses, and EDSS assessments were performed after regular intervals of 3 months. The course of disease was followed for 5 years, even if the patients terminated the treatment during follow-up.

To detect possible pre-treatment differences in the patient population between the $M X 1_{\text {high }}$ and the $M X 1_{\text {low }}$ groups, we compared age and EDSS at baseline, elapsed time between diagnosis of definite MS and study onset, as well as the number of relapses 1 year prior to the study, applying the two-sample two-tailed $t$-test assuming equal variances with significance level alpha $=0.05$. The sex ratio and the number of patients per administered IFN-beta formulation were compared between the cohorts with Fisher's exact test at the alpha $=0.05$ level of significance. Furthermore, these patient parameters were checked for correlation with the baseline expression levels of the pathway genes by calculating the Pearson correlation coefficient. Because of multiple testing, only correlation $P$-values below 0.001 were considered statistically significant.

We investigated the clinical relevance of endogenous IFN-like activity on the long-term course of disease by evaluating the progression of EDSS and the number of relapses during a 5-year follow-up period after the start of IFN-beta therapy. Mean EDSS scores and the cumulative number of relapses have been compared between the two patient groups using the $t$-test with Welch correction. Additionally, we compared the relapse-free survival during the follow-up by constructing rightcensored Kaplan-Meier curves with PASW Statistics. The relapse-free survival curves for the $M X 1_{\text {high }}$ patient group and the $M X 1_{\text {low }}$ patient group have been tested for significant deviation by computing the log-rank test and the Cox proportional hazard ratio.

\section{Results}

\section{Interferon pathways}

We identified 46 genes that have redundantly been described in the literature to play a role in the pathways inducing IFN-beta transcription and the pathway that is activated by IFN-beta [34-44]. We complemented the list of genes with 10 genes that have repeatedly been reported as ISGs, including MX1 (Additional file 1). The genes, and their interactions within the IFN-beta induction pathways and the IFN-beta effector pathway, will be summarized briefly below. 
The expression of IFN-beta is physiologically induced after recognition of viral, bacterial or degraded endogenous nucleic acids by cellular receptors. This is mediated by the activation of two main pathways. The endosomal Toll-like receptors (TLRs) TLR7, -8 and -9 activate a $M y D 88$-dependent pathway with the signal transducers IRAK4, IRAK1 and TRAF6. The latter leads via MAP3K7 (TAK1) to the phosphorylation of the kinases $I K K$, MAPK8 (JNK) and MAPK14 (p38), resulting in the activation of the transcription factors NFkappaB, JUN and $A T F 2$, respectively. The other main pathway is initiated by the endosomal TLR3, as well as by the cytoplasmic receptors IFIH1 (MDA5), DDX58 (RIG-I) and ZBP1. They induce the phosphorylation of the IFN regulatory factors (IRF) IRF3 and IRF7 via TRAF3 and TBK1/IKKepsilon. These IRFs then homodimerize or heterodimerize and translocate to the nucleus. The cell membranebound TLR4 can initiate both the MyD88 pathway and the TRAF3 pathway. The activated TFs bind to the promoter region of IFN-beta (IFNB1) and initiate its transcription [51]. Several cross-bridges between the $M y D 88$ and the TRAF3 pathway exist [34-38,40,41,43,44].

Endogenously produced IFN-beta and exogenously injected IFN-beta bind to the IFN-alpha/beta receptor. This receptor comprises the subunits IFNAR1 and IFNAR2 with the associated kinases TYK2 and JAK1. Once activated, the IFNAR complex phosphorylates STAT1 and STAT2, which heterotrimerize with IRF9 to form the ISGF3 complex (JAK/STAT pathway). ISGF3 translocates to the nucleus and binds the interferonstimulated response element (ISRE), a DNA motif that can be found in the regulatory region of many ISGs. Activated STATs also form homodimers and heterodimers acting as TFs independent of IRF9 [35-40,42,43]. The activation of STATs in response to IFN-beta was shown to differ among leukocyte subsets, with STAT1 being mainly activated in monocytes and CD8+ T cells [52].

There are several positive and negative feedback loops in the type I IFN-related pathways. Many receptors, signal transducers and TFs within the IFN-beta induction pathway are themselves induced by IFNs, thereby amplifying IFN responses, for example IRF7, DDX58 and IFIH1 [37,39]. As a negative feedback, the JAK/STAT pathway stimulates the transcription of several suppressor of cytokine signaling (SOCS) genes, which act as inhibitors on TLRs and JAKs, and target the IFNAR complex for proteasomal degradation [35].

\section{Patient characteristics}

The 61 RR-MS patients (42 females, 19 males) in our study were between 18 and 63 years of age at the start of treatment with IFN-beta. The time elapsed between diagnosis of MS and therapy initiation was less than
9 months for 53 patients and between 25 and 134 months for the remaining 8 patients. At the time of blood sampling, there was no evidence of an ongoing infection based on clinical signs and laboratory testing. Over the complete 5-year observation period, 55 of the 61 patients have been followed at our clinic and 41 patients continuously received the prescribed IFN-beta therapy. Eleven of the 61 patients had relatively elevated baseline $M X 1$ expression levels. They were allocated to the $M X 1_{\text {high }}$ cohort, while the remaining 50 patients were allocated to the $M X 1_{\text {low }}$ cohort. There was no significant difference between the two patient groups in terms of age, sex ratio and baseline EDSS. The time between diagnosis and study onset, as well as the number of relapses during the year prior to the study, were comparable (Table 1). No significant correlation between any of these patient characteristics and the pre-treatment expression levels of the 56 pathway genes could be observed.

\section{Expression levels and dynamics dependent on MX1 status} For comparison of the PBMC gene expression between the two patient groups and between the two timepoints, only patients who received IFN-beta sc were considered (see Methods). Of these individuals, 7 patients were in the $M X 1_{\text {high }}$ group and 30 patients were in the $M X 1_{\text {low }}$ group. After 1 month of therapy, the $M X 1$ expression levels of the $M X 1_{\text {high }}$ cohort remained relatively unchanged whereas $M X 1_{\text {low }}$ patients showed a significant induction up to the levels of the $M X 1_{\text {high }}$ group (Figure 1). Similar expression patterns were observed for other ISGs.

When comparing the mean baseline expression of the 56 pathway genes between both cohorts, 11 genes were expressed in the $M X 1_{\text {high }}$ group at a significantly higher level. Elevated transcript levels were found for cytoplasmic receptors (for example, IFIH1), TFs (for example, IRF7, IRF9 and STAT1) and, as expected, most ISGs (Figure 2 and Additional file 3). Other receptors, such as TLR7 and TLR8, as well as the transcription factor STAT2, also showed elevated expression in $\mathrm{MX} 1_{\text {high }}$ patients, but with $P$-values $>0.01$. We found no significant difference in expression at the mRNA level for natural IFN-beta (IFNB1) and its receptors IFNAR1 and IFNAR2.

During the first month of IFN-beta sc therapy, the $M X 1_{\text {high }}$ group showed no significant change in the expression of the IFN-beta-related pathway genes (Additional file 4). In contrast, 25 of the 56 genes were found to be significantly modulated in $\mathrm{MX}_{\text {low }}$ patients, with 23 genes being upregulated and two genes (TLR9 and IRAK4) being downregulated in response to therapy (Figure 3). The upregulated genes comprised all the 11 genes that were significantly elevated in the $M X 1_{\text {high }}$ patient group at baseline. 
Table 1 Clinical and demographic parameters of the two patient cohorts

\begin{tabular}{|c|c|c|c|}
\hline Demographic and clinical characteristics & $M X 1_{\text {high }}(n=11)$ & $M X 1_{\text {low }}(n=50)$ & $P$-value \\
\hline Age at study onset (years) & $34.8 \pm 8.7$ & $38.3 \pm 10.4$ & 0.312 \\
\hline Time from diagnosis to therapy (months) & $2.4 \pm 2.4$ & $11.6 \pm 26.6$ & 0.258 \\
\hline EDSS at baseline & $2.0 \pm 1.5$ & $1.7 \pm 1.1$ & 0.510 \\
\hline Number of relapses during the year prior to study onset & $1.2 \pm 0.4$ & $0.9 \pm 0.6$ & 0.172 \\
\hline Gender (women/men) & $8 / 3$ & $34 / 16$ & 1.000 \\
\hline Type of IFN-beta administered (1a im/1a sc/1b sc) & $4 / 3 / 4$ & $20 / 9 / 21$ & 0.826 \\
\hline
\end{tabular}

Mean \pm standard deviation are given for age, disease duration and expanded disability status scale (EDSS) at study onset as well as for the number of relapses in the year prior to treatment. These parameters were tested for differences between patients with low and high endogenous IFN-like activity (patient groups $M X 1_{\text {low }}$ and $M X 1_{\text {high }}$ ) applying the $t$-test. For the sex ratio and the administered IFN-beta products, the absolute patient numbers are given and $P$-values as calculated by Fisher's exact test. im, intramuscular; sc, subcutaneous.

After 1 month of therapy, there was no difference in expression of the genes between the two patient groups (Additional file 5).

The differences in gene expression over time and between the cohorts are summarized in Figure 4 and in Additional file 3. The pathway visualizations are available from the authors as Cytoscape session files upon request.

\section{Real-time PCR analysis of differential gene expression}

Of the 56 pathway genes, 14 were re-measured by realtime PCR. Overall, the PCR analyses confirmed the microarray results. Despite the long storage of the samples, the mRNA levels obtained by the two techniques correlated significantly for all genes except TLR9 (Additional file 2). Of the $11 \mathrm{MX} 1_{\text {high }}$ patients, nine were contained in the PCR data. As in the microarray data, they also showed a higher pre-treatment expression of MX1 (Ct values $<26.7$ ) than all $M X 1_{\text {low }}$ patients in the PCR data. In the subgroup of patients treated with IFN-

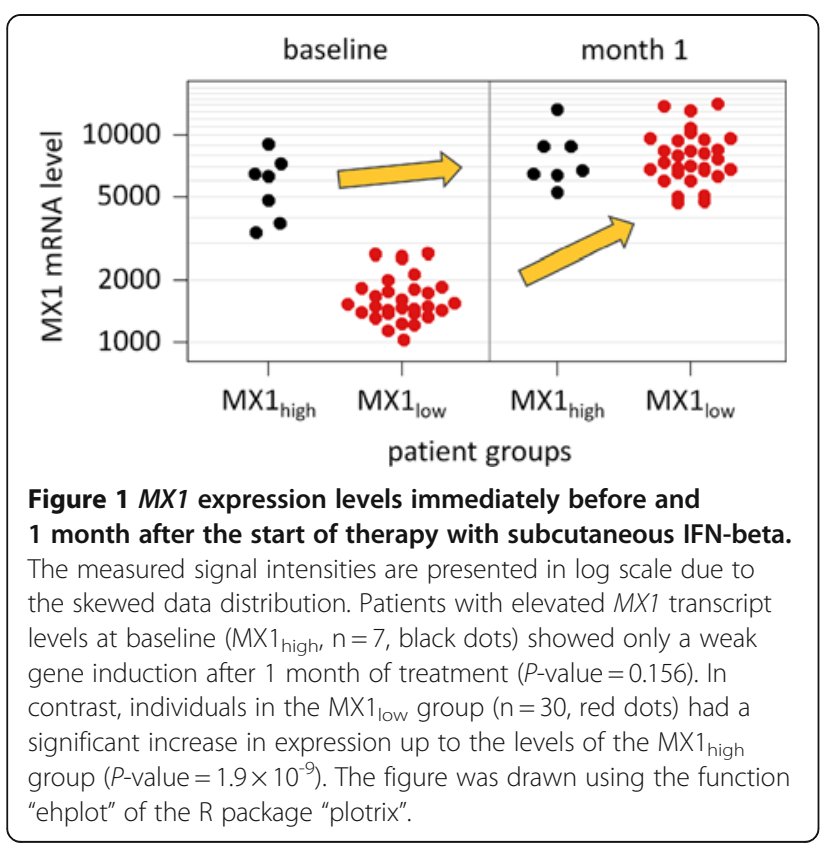

beta sc, there were five $M X 1_{\text {high }}$ patients and $21 \mathrm{MX} 1_{\text {low }}$ patients. Although sample sizes were smaller, the expression differences observed in the Affymetrix microarray data were also seen in the real-time PCR data: at baseline, MX1, MYD88, RSAD2, STAT1 and STAT2 were expressed at higher levels in $M X 1_{\text {high }}$ patients, and they were significantly upregulated during therapy only in the $\mathrm{MX} 1_{\text {low }}$ cohort (Additional file 3).

\section{ISGF3 is a central regulator of type I IFN signaling}

Predicted TFBS for ISGF3 were found in the promoter regions of 10 out of the 56 pathway genes (Additional file 3). There was a marked overlap when comparing the genes putatively regulated by the ISGF3 complex with those genes overexpressed in $M X 1_{\text {high }}$ patients at baseline. The receptors $I F I H 1, D D X 58$ and $Z B P 1$, two ISGs (CXCL10, ISG20), IFNB1, the pathway inhibitors SOCS1 and SOCS3, as well as the TFs IRF7 and STAT2 contained predicted DNA-binding sites for ISGF3 in their promoter region. In this way, ISGF3 forms multiple positive and negative regulatory feedback loops. We conclude from this analysis that a higher expression and activity of the ISGF3 complex is most likely responsible for the higher endogenous type I IFN-like activity seen in a subset of patients.

\section{Stability of MX1 mRNA expression}

In the time-course expression data by Karlovich et al. [50], we observed that approximately one-fifth of the healthy subjects showed relatively elevated $M X 1$ transcript levels in the blood, which is very similar to the proportion we ascertained for the MS patients in our study. Differential endogenous type I IFN-like activities therefore seem to be not exclusive for MS patients, and may rather demonstrate individual variation of innate immunity. Moreover, high $M X 1$ expression at study onset correlated strongly with the levels measured after 6 months $(P=0.00003)$. This revealed that the natural course of $M X 1$, and thus the IFN-beta-like activity, is stable over time. ANOVA confirmed this result; the variance in the data could be explained best by intersubject 


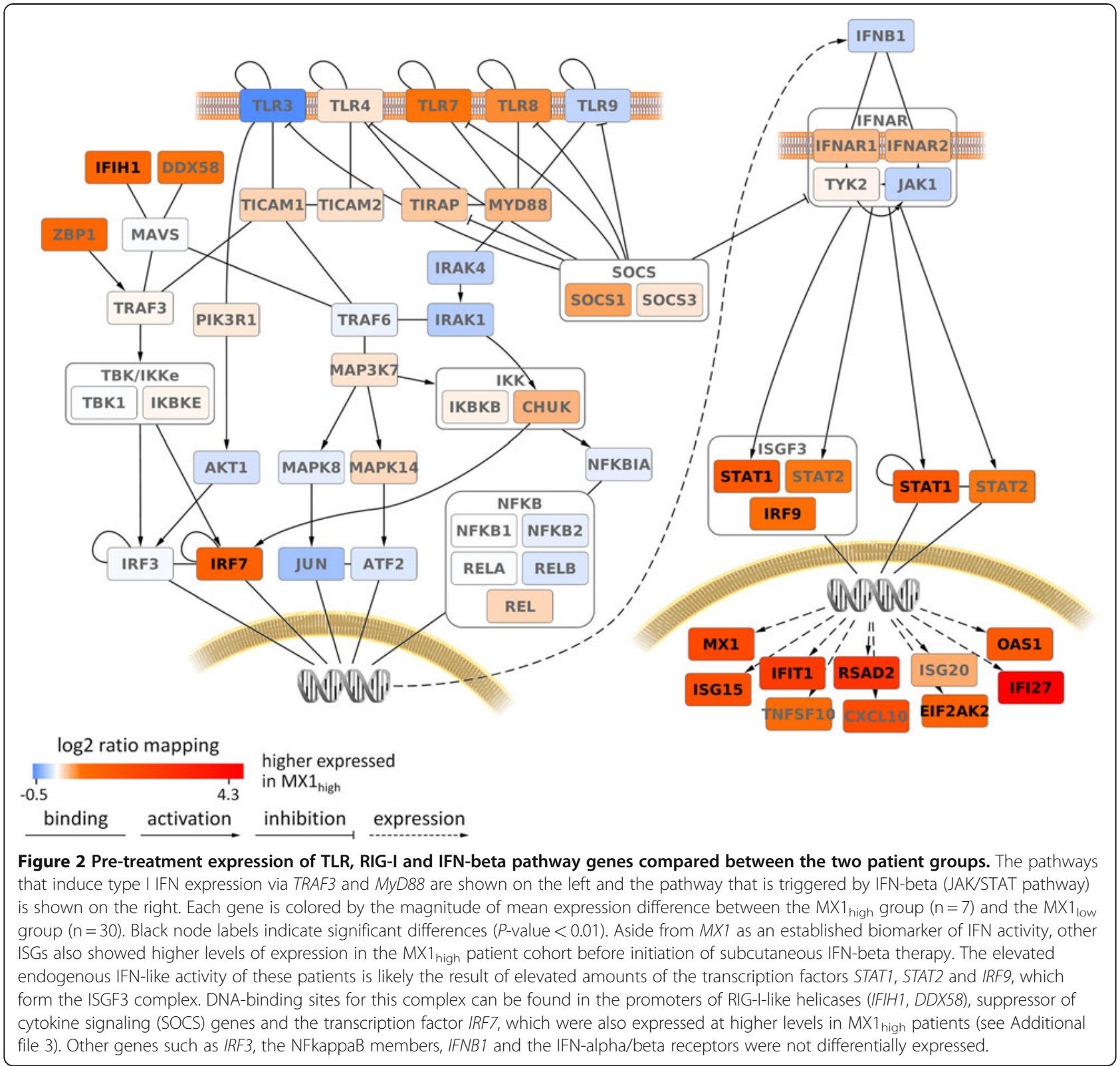

differences $\left(P=3.6 \times 10^{-12}\right)$, while the effects of age and gender were not significant. The $M X 1$ expression data of the 22 healthy individuals are shown in Additional file 6.

\section{MX1 status and clinical outcome}

To evaluate whether the endogenous IFN-beta-like activity is associated with individual disease progression and IFN-beta therapy outcome, we compared the $M X 1_{\text {high }}$ group and the $M X 1_{\text {low }}$ group in terms of EDSS and relapses as clinical measures of the longterm course of disease. The EDSS-defined changes of disability differed considerably between the individual patients. However, when comparing the mean EDSS of the two patient groups, the course of progression was very similar (Figure 5). After 5 years, the EDSS of the $M X 1_{\text {high }}$ cohort and the $M X 1_{\text {low }}$ cohort had risen, on average, by 0.85 and 0.92 points, respectively. The mean 5 -year relapse rate also did not differ significantly between the $M X 1_{\text {high }}$ group (1.6) and the $M X 1_{\text {low }}$ group (1.7) (Figure 5). Accordingly, the Kaplan-Meier curves for relapse-free survival of both groups were very similar in their decline, especially during the first 2 years (Figure 6). For the complete 5-year follow-up period the calculated log-rank statistic $(P=0.424)$ and the hazard ratio $(0.68$, confidence interval: 0.27 to 1.76 ) showed no significant difference in the proportion of relapse-free patients between the cohorts. 


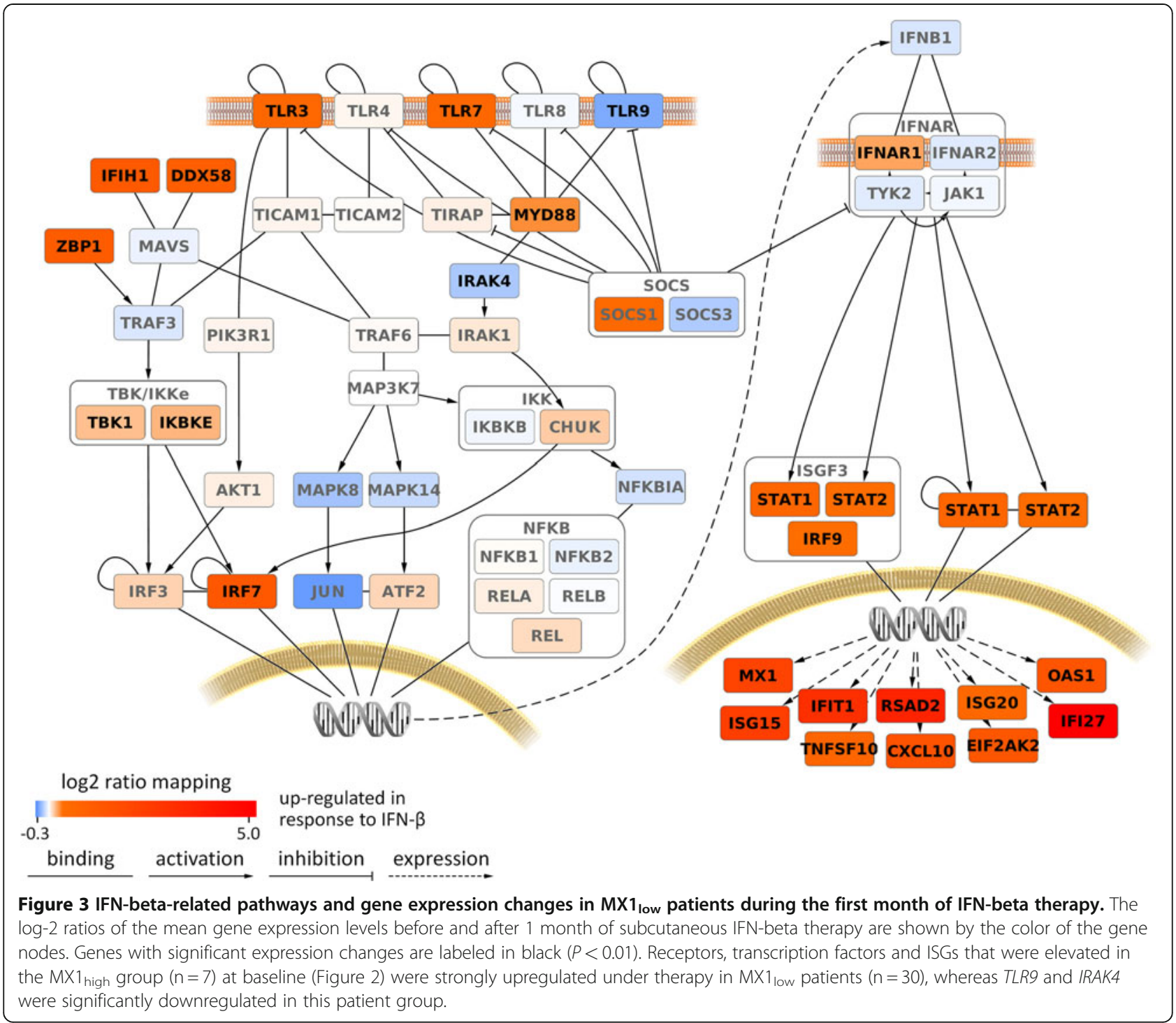

However, when comparing the relapse rate between $M X 1_{\text {high }}$ patients and $M X 1_{\text {low }}$ patients separately for the three different drug formulations, we could observe significant differences (Additional files 7 and 8). In the subgroup of patients treated with IFN-beta-1a im, all the $M X 1_{\text {high }}$ patients $(n=4)$ were free of relapses over 5 years whilst the $M X 1_{\text {low }}$ patients $(n=17)$ experienced 1.6 relapses on average. In contrast, within the patient group receiving IFN-beta-1b sc, the $M X 1_{\text {high }}$ cohort $(n=3)$ had a much higher average number of relapses than the $M X 1_{\text {low }}$ cohort $(\mathrm{n}=20)(2.7$ versus 1.3$)$. MX1 $1_{\text {high }}$ patients and $M X 1_{\text {low }}$ patients had similar changes on the EDSS for each of the drug preparations. However, patients receiving IFN-beta im were diagnosed and treated at an earlier stage of the disease (mean baseline EDSS: 1.0) than those receiving IFN-beta sc (mean baseline EDSS: 2.3), and they were also more stable during the followup period.

\section{Discussion}

Our results revealed that approximately one-fifth of MS patients show an elevated endogenous type I IFN-like activity prior to IFN-beta treatment and that this phenomenon can be explained by a higher expression and activity of the ISGF3 complex. This complex comprises the TFs STAT1, STAT2 and IRF9, and is not just a key regulator of ISGs, but is also important to induce diverse genes involved in IFN regulation and signaling $[37,53]$. Therefore, patients with high levels of the IFN activity biomarker $M X 1$ ( $M X 1_{\text {high }}$ cohort) also showed high levels of other ISGs and several pathway genes, which in turn can control type I IFN transcription, forming positive feedback loops [39]. The link between ISGF3 and IFN-like expression signature is supported by the results of the TFBS analysis, which revealed that the list of ISGF3 target genes to a certain extent resembles the list of genes elevated in $M X 1_{\text {high }}$ patients. Our study is 


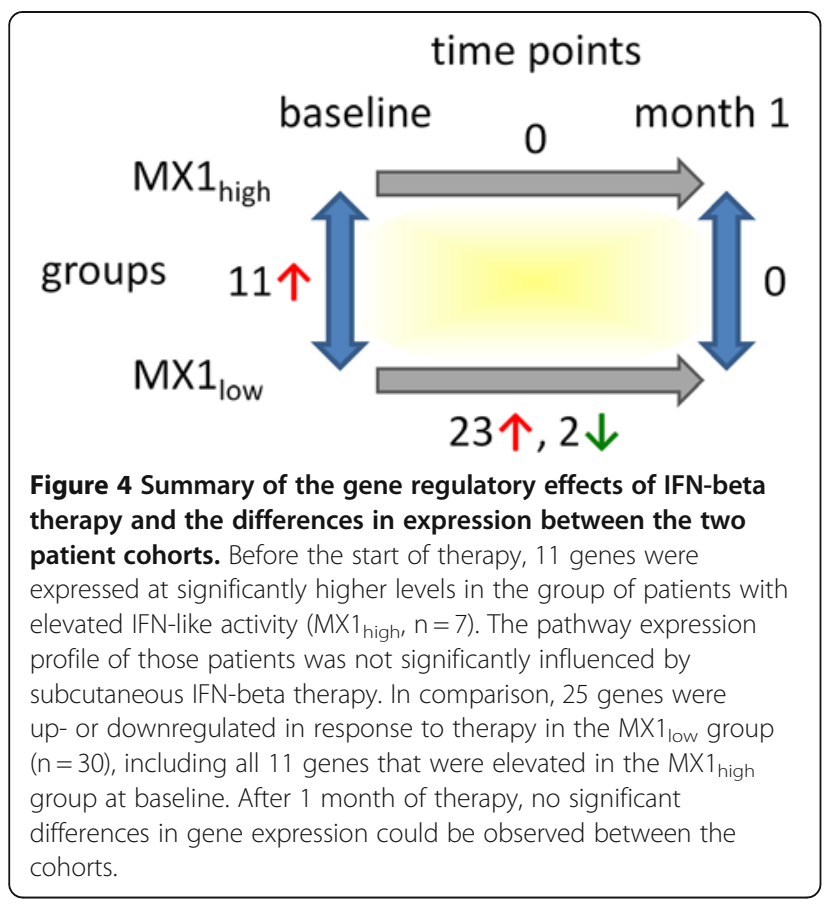

limited by the fact that we measured the gene expression in PBMCs at the mRNA level, which does not necessarily correlate with the amounts of active proteins due to post-transcriptional gene regulation, for example by microRNAs $[54,55]$. However, earlier studies at the protein level have already shown that the phosphorylation status of the ISGF3 component STAT1 correlates with the ISG expression in MS patients [31].

We observed increased pre-treatment levels of the single-stranded RNA receptors TLR7 and TLR8 in $M X 1_{\text {high }}$ patients compared with $M X 1_{\text {low }}$ patients. This may also be mutually linked to an overactive JAK/STAT pathway in the $M X 1_{\text {high }}$ group of patients. The expression differences for these two TLR receptors did not reach the significance threshold $(P$-values $<0.01)$. However, this may be due to the fact that a different Affymetrix chip type (U133 Plus 2.0) was used for the samples of patients treated with IFN-beta-1a sc than for the others (U133 A and B). For some genes, including these two TLRs, differences in the custom probesets between both chip types led to increased variation in the data and thus somewhat higher $P$-values (Additional files 2 and 3).

Similar to our patients, we also found a relatively high type I IFN-like activity in a subset of healthy individuals, and $M X 1$ levels in the respective individuals remained elevated over the whole study period of 6 months. Therefore, independent of MS disease, some people seem to have a constitutive, robust signature of immune activation, while others do not. It has been described that the immune defense program

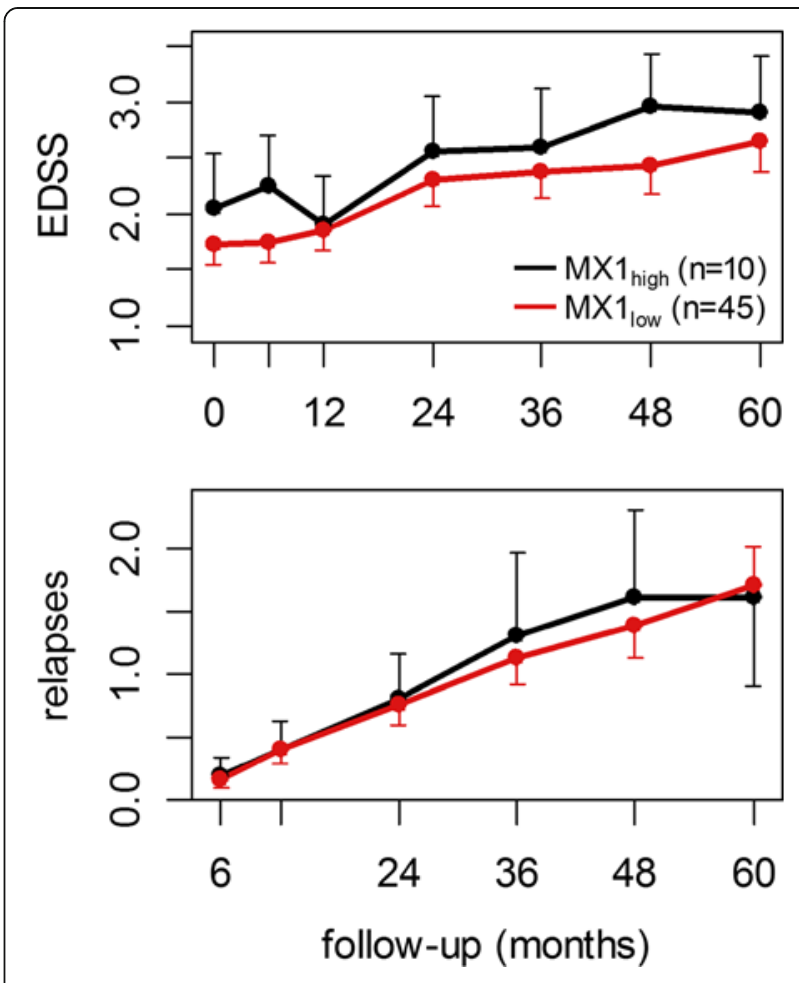

Figure 5 Long-term progression of disability and cumulative number of relapses. Mean time-courses are shown in black for the $M X 1_{\text {high }}$ group and in red for the $M X 1_{\text {low }}$ group. The change of disability according to the expanded disability status scale (EDSS) as well as the relapse rate during the 5-year follow-up period were similar for both cohorts (Welch's $t$-test $P$-values $>0.2$ ). $M X 1_{\text {high }}$ patients presented on average a slightly worse EDSS at baseline, and this nonsignificant difference persisted during the treatment. Error bars represent standard errors.

is, in general, slightly more active in MS [26,56]. However, we could not evaluate this aspect, since we used external data on the whole blood of healthy subjects where it is difficult to compare the absolute mRNA signals with our PBMC data on MS patients. Still, the data on the healthy subjects provided strong evidence that the observed increased IFN-beta-like activities are not just the result of short-term hidden viral infections. This is further supported by our finding that the IFN-beta gene itself (IFNB1) was not expressed at higher levels in the $M X 1_{\text {high }}$ group as would be expected during an activated antiviral response (hence we used the term "IFN-like activity"). However, IFNB1 transcript levels were virtually undetectable in our Affymetrix microarray data (Additional file 2), and we have not measured them with real-time PCR. Others have used more sensitive methods and could detect an elevated endogenous IFN-beta expression in PBMCs at baseline in IFNbeta therapy non-responders [29], which they believed to be causal for an elevated type I IFN signature [28]. 


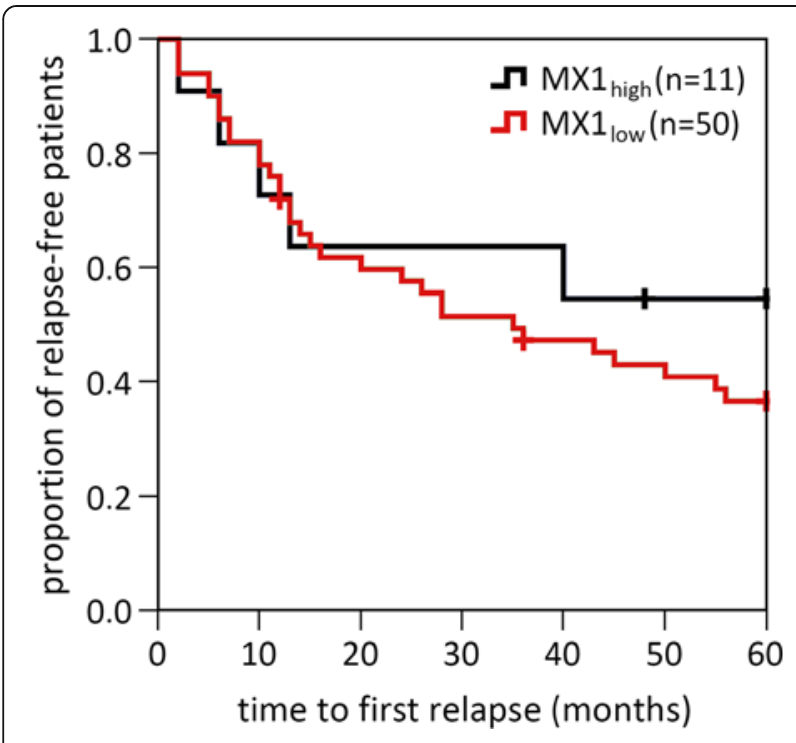

Figure 6 Kaplan-Meier survival curves for patients with low and high endogenous IFN-like activity. The Kaplan-Meier curves show the proportion of relapse-free patients in the $M X 1_{\text {high }}$ group (black curve) and in the $M X 1_{\text {low }}$ group (red curve). Vertical dashes indicate right-censored patients that dropped out of clinical surveillance $(n=3)$. In particular, during the first 18 months after therapy initiation, the proportions of relapse-free patients declined in a similar manner for both cohorts. Overall, the difference between both patient groups was not significant according to the log-rank test $(P=0.424)$ and the hazard ratio $(0.684$, confidence interval: 0.266 to 1.761$)$

We could not confirm these conclusions with the data from our study.

During IFN-beta sc therapy, patients with relatively high ISG expression at baseline showed no significant modulation in the expression of the genes involved in IFN-beta-related pathways. In contrast, patients in the $M X 1_{\text {low }}$ group showed a strong gene induction after 1 month of treatment (Figure 4). The congruency between the set of genes primarily elevated in $\mathrm{MX}_{\text {high }}$ patients and the genes induced by therapeutic IFN-beta in $M X 1_{\text {low }}$ patients was striking. Possibly, higher levels of $M X 1$ and its co-expressed genes might be observed during therapy in both patient groups, if the blood expression is measured in the first $12 \mathrm{~h}$ after an IFNbeta injection $[22,23]$. Moreover, since we only measured the changes in mRNA expression, additional experiments on the protein level are needed to further investigate specific issues, for example the correlation between the amounts of STAT transcripts and the amounts of phosphorylated STAT proteins. Similarly, while we observed no significant blood expression differences for IFN-alpha/beta receptors, we can only speculate about the amounts and cell type-specific localization of the corresponding proteins. Comabella et al. $[28,29]$ used flow cytometry to measure the expression of IFNAR1 on monocytes. In patients with ongoing disease activity despite IFN-beta treatment, they found a higher baseline expression of this receptor [28,29]. Dupont et al. [57] showed, using in vitro experiments with Jurkat cells, that the surface expression of IFNAR1 decreases after IFNbeta application and that the cells were subsequently unresponsive to further IFN-beta treatment for some hours. We may miss such findings in the PBMC population. Additionally, receptor polymorphisms, or alternatively spliced transcripts of these receptors, could play a role. Different splice variants of the IFNAR2 receptor show different biologic responses to endogenous and exogenous IFN-beta and there is evidence that IFN-beta immunogenicity may be, in part, related to IFNAR2 isoform expression [58]. However, since NAbs were not routinely measured for our patients, we were not able to investigate this possible association of IFNAR expression and NAbs.

A further interesting observation was that the levels of TLR3 and -7 were significantly upregulated, while TLR 9 was significantly downregulated, in $M X 1_{\text {low }}$ patients during therapy. Pre-treatment levels of TLR3 were somewhat lower for $\mathrm{MX}_{\text {high }}$ patients $(P=0.059)$, but the expression also strongly increased within 1 month of IFN-beta administration in this patient group $(P=0.016)$. TLR7 is engaged in the maturation of dendritic cells, which play a role in bridging innate and adaptive immunity [59]. Upregulation of TLR7 on dendritic cells by IFN-beta has been shown to inhibit the differentiation of Th17 cells [60], which have been recognized as proinflammatory lymphocytes involved in MS pathogenesis [61]. TLR-mediated signaling and IFN signaling is counter-regulated by SOCS1, the mRNA levels of which were upregulated in the $M X 1_{\text {low }}$ patient group during therapy $(P=0.017)$. A genetic risk factor for MS has been identified in the SOCS1 promoter region recently [62].

We addressed the clinical importance of endogenous IFN-like activity and its prognostic power to predict disease progression and clinical response to IFN-beta therapy, an issue that has been debated with contradictory results [27-33]. Some studies suggest that baseline overexpression of ISGs may indicate pre-activated pathways in a way that IFN injections are less effective, which may explain suboptimal therapeutic responses $[28,30]$. However, a correlation between an elevated endogenous type I IFN signature and a worse course of disease could not be confirmed in our study, even though the $M X 1_{\text {high }}$ patient group showed a much weaker biologic response within the first treatment month, at least regarding the mRNA levels of the 56 examined pathway genes. Over the 5-year follow-up period, neither the average number of relapses nor the increase in EDSS were significantly different between patients with low and high baseline $M X 1$ levels. On the contrary, in a study 
by van der Voort et al. [32], high $M X 1$ mRNA expression was predictive of a longer time to a first new relapse. However, less than half of their patients that were initially therapy-naive started an immunomodulatory treatment during follow-up, and the baseline $M X 1$ levels did not differ significantly between responder status groups for the subset of treated patients. The beneficial effect of an increased IFN-like activity thus may be observed primarily in untreated patients. This is supported by the results of Hesse $e t$ al. [33], who found an indirect negative correlation between the number of active lesions and the blood expression of MX1 in untreated patients. We hypothesize that a higher endogenous IFN-like activity does not imply IFN-beta therapy non-response, but might be positive in untreated patients. Nevertheless, the question remains whether MX1 $1_{\text {high }}$ patients could benefit more from other treatments than from IFN-beta.

Our analysis revealed some differences in the relapse rates of the $M X 1_{\text {high }}$ group and the $M X 1_{\text {low }}$ group when analyzing the data for each IFN-beta drug preparation separately. However, this result must be interpreted with caution for two reasons: firstly, for each of the three therapy regimes, the number of patients per MX1 group is relatively small; and secondly, we did not systematically test for NAbs after long-term treatment, but their appearance might have led to reduced therapeutic benefits in some of the patients. In our data, $M X 1_{\text {high }}$ patients treated with once weekly $30 \mu \mathrm{g}$ IFN-beta-1a im experienced no relapse during the 5 -year follow-up period, while $\mathrm{MX} 1_{\text {high }}$ patients receiving high-frequent high-dose IFN-beta-1b sc experienced, on average, 2.7 relapses. It is tempting to speculate about the reasons for this discrepancy. One possible explanation might be that only intermediate levels of IFN-beta are beneficial. Patients with low inherent type I IFN-like activity might need frequent IFN-beta therapy, whereas in $M X 1_{\text {high }}$ patients such a more intense IFN administration may not yield the desired effects, and possibly raises the chance of $\mathrm{NAb}$ development. An alternative explanation might be found in the disease duration. Patients treated with IFNbeta-1a im were, according to the EDSS at study onset, in an earlier stage of MS, and it is thus conceivable that a higher endogenous IFN signature combined with IFN-beta treatment might be preferentially favorable early in disease. Therapy-specific studies with larger cohorts are needed to test whether $M X 1$ has the potential to support the choice of IFN-beta drug preparation for the individual patient. Such further investigations must carefully consider the issues of dosage and frequency of IFN-beta administration, and should take the issue of NAbs into account.

\section{Conclusions}

In conclusion, an elevated type I IFN-like activity was observed in the blood of a subset of MS patients as well as a subset of healthy individuals. We showed for the healthy subjects that this gene expression signature is robust over time and is therefore probably not the result of a transient latent infection. For the MS patients we demonstrated that the expression of genes involved in IFN-related pathways is strongly induced by IFN-beta therapy in those patients with low endogenous IFN-like activity, but is only weakly induced in patients with high endogenous IFN-like activity. The baseline gene expression pattern characteristic for the latter group resembles the pattern induced by therapeutic IFN-beta. While the natural IFN-beta and its receptors were expressed at similar mRNA levels in both groups, genes of the ISGF3 transcription factor complex were expressed significantly more in patients with an elevated endogenous IFN-betalike signature. Our TFBS analysis led us to the hypothesis that the activity of the ISGF3 complex is crucial for the individually different activation status of the IFN pathways. Our study, which was limited by a relatively small sample size, did not confirm that the IFN signature prior to treatment with IFN-beta is predictive of the individual long-term course of MS, but different outcomes were observed for different drug formulations. This deserves further investigation with more sensitive and cell type-specific analyses on the RNA and protein level.

\section{Additional files}

\begin{abstract}
Additional file 1: References to review articles addressing the genes involved in IFN-related pathways.

Additional file 2: This table provides the preprocessed microarray data of the 56 pathway genes for all 61 patients before therapy initiation (baseline) and after 1 month of IFN-beta treatment.

Additionally, it contains the real-time PCR data for 14 of these genes that were obtained for 44 of the patients.

Additional file 3: Genes involved in the pathways regulating IFNbeta expression and in the pathway that is activated by IFN-beta. Mean microarray signal intensities, calculated Wilcoxon test $P$-values as well as ISGF3 DNA-binding site information are provided for all genes. Moreover, for 14 of the genes, the results of the real-time PCR analysis are also shown.
\end{abstract}

Additional file 4: IFN-beta-related signaling pathways displaying the pharmacological effects on gene expression for the $M X 1_{\text {high }}$ patient group after 1 month of treatment with IFN-beta sc.

Additional file 5: Expression of TLR, RIG-I and IFN-beta pathway genes compared between both cohorts at 1 month after start of IFN-beta sc therapy.

Additional file 6: The natural course of $M X 1$ mRNA expression is shown for 22 healthy individuals over a period of 6 months.

Additional file 7: Comparison between both cohorts in terms of EDSS changes and cumulative number of relapses during a 5-year observation period displayed separately for each IFN-beta drug preparation.

Additional file 8: The proportion of relapse-free patients during 5 years of follow-up for both patient groups, dependent on the administered IFN-beta drug preparation.

\section{Abbreviations}

ANOVA: Analysis of variance; CDF: Chip definition file; Ct: Cycle threshold; EDSS: Expanded disability status scale; IFN: Interferon; IFNAR: IFN-alpha/beta 
receptor; IL: Interleukin; im: Intramuscular; IRF: IFN regulatory factor; ISG: Interferon-stimulated gene; ISGF3: IFN-stimulated gene factor 3; ISRE: Interferon-stimulated response element; MS: Multiple sclerosis; MX1: Myxovirus resistance protein 1; NAb: Neutralizing antibody; PBMC: Peripheral blood mononuclear cells; PCR: Polymerase chain reaction; RR-MS: Relapsing-remitting multiple sclerosis; sc: Subcutaneous; SOCS: Suppressor of cytokine signaling; STAT: Signal transducer and activator of transcription; TFBS: Transcription factor binding sites; TF: Transcription factor; TLR: Toll-like receptor.

\section{Competing interest}

UKZ received research support as well as speaking fees from Bayer, Biogen Idec, Merck Serono, Novartis, Sanofi-Aventis, and Teva. MH received speaking fees from Bayer HealthCare. The other authors declare that they have no competing interests.

\section{Acknowledgements}

This work was co-funded by the German Federal Ministry for Education and Research (NBL3 program, reference 01 ZZ 0103) and by the United Europeans for the development of PHArmacogenomics in Multiple Sclerosis consortium (UEPHA*MS). The Affymetrix microarray experiments were funded by Bayer HealthCare, Merck Serono and Biogen Idec.

\section{Authors' contributions}

UKZ and HJT inspired and directed the work. OK, CF, CH and UKZ were responsible for patient care and clinical documentation. The lab experiments were performed by BKP and DK. AH and $\mathrm{MH}$ carried out the analysis and interpretation of the data, drafted the paper and prepared all tables and figures. CA, BKP and DK assisted in compiling the literature as well as in interpreting the results and contributed to the writing of the manuscript. The paper was corrected, improved and completed by HJT and UKZ, and all authors read and approved the final version.

Received: 23 January 2012 Accepted: 22 June 2012

Published: 22 June 2012

\section{References}

1. Compston A, Coles A: Multiple sclerosis Lancet 2008, 372:1502-1517.

2. Sawcer S, Hellenthal G, Pirinen M, Spencer CC, Patsopoulos NA, Moutsianas L, Dilthey A, Su Z, Freeman C, Hunt SE, Edkins S, Gray E, Booth DR, Potter SC, Goris A, Band G, Oturai AB, Strange A, Saarela J, Bellenguez C, Fontaine B, Gillman M, Hemmer B, Gwilliam R, Zipp F, Jayakumar A, Martin R, Leslie S, Hawkins S, Giannoulatou E, et al:: Genetic risk and a primary role for cellmediated immune mechanisms in multiple sclerosis. Nature 2011, 476:214-219.

3. Sospedra M, Martin R: Immunology of multiple sclerosis. Annu Rev Immunol 2005, 23:683-747.

4. Hauser SL, Oksenberg JR: The neurobiology of multiple sclerosis: genes, inflammation, and neurodegeneration. Neuron 2006, 52:61-76.

5. McFarland HF, Martin R: Multiple sclerosis: a complicated picture of autoimmunity. Nat Immunol 2007, 8:913-919.

6. Hafler DA, Slavik JM, Anderson DE, O'Connor KC, De Jager P, Baecher-Allan C: Multiple sclerosis. Immunol Rev 2005, 204:208-231.

7. Koch $M$, Kingwell E, Rieckmann $P$, Tremlett $H$ : The natural history of secondary progressive multiple sclerosis. J Neurol Neurosurg Psychiatry 2010, 81:1039-1043.

8. Zettl UK, Stuve O, Patejdl R: Immune-mediated CNS diseases: A review on nosological classification and clinical features. Autoimmun Rev 2012, 11:167-173.

9. Farrell RA, Giovannoni G: Current and future role of interferon beta in the therapy of multiple sclerosis. J Interferon Cytokine Res 2010, 30:715-726.

10. Vosoughi R, Freedman MS: Therapy of MS. Clin Neurol Neurosurg 2010, 112:365-385.

11. Mendes A, Sa MJ: Classical immunomodulatory therapy in multiple sclerosis: how it acts, how it works. Arq Neuropsiquiatr 2011, 69:536-543.

12. Paap BK, Hundeshagen A, Hecker M, Zettl UK: An inventory of short term and long term changes in gene expression under interferon beta treatment of relapsing remitting MS patients. Curr Pharm Des. in press.

13. Rudick RA, Polman $\mathrm{CH}$ : Current approaches to the identification and management of breakthrough disease in patients with multiple sclerosis. Lancet Neurol 2009, 8:545-559.
14. Graber JJ, Dhib-Jalbut S: Biomarkers of disease activity in multiple sclerosis. J Neurol Sci 2011, 305:1-10.

15. Vandenbroeck K, Comabella M: Single-nucleotide polymorphisms in response to interferon-beta therapy in multiple sclerosis. $J$ Interferon Cytokine Res 2010, 30:727-732.

16. Hecker M, Paap BK, Goertsches RH, Kandulski O, Fatum C, Koczan D, Hartung HP, Thiesen HJ, Zettl UK: Reassessment of blood gene expression markers for the prognosis of relapsing-remitting multiple sclerosis. PLoS One 2011, 6:e29648.

17. Grossberg SE, Oger J, Grossberg LD, Gehchan A, Klein JP: Frequency and Magnitude of Interferon beta Neutralizing Antibodies in the Evaluation of Interferon beta Immunogenicity in Patients with Multiple Sclerosis. J Interferon Cytokine Res 2011, 31:337-344.

18. Kappos L, Clanet M, Sandberg-Wollheim M, Radue EW, Hartung HP, Hohlfeld R, Xu J, Bennett D, Sandrock A, Goelz S: Neutralizing antibodies and efficacy of interferon beta-1a: a 4-year controlled study. Neurology 2005, 65:40-47.

19. Killestein J, Polman $\mathrm{CH}$ : Determinants of interferon beta efficacy in patients with multiple sclerosis. Nat Rev Neurol 2011, 7:221-228.

20. Hesse D, Sellebjerg F, Sorensen PS: Absence of MxA induction by interferon beta in patients with MS reflects complete loss of bioactivity. Neurology 2009, 73:372-377.

21. Goertsches RH, Hecker M, Zettl UK: Monitoring of multiple sclerosis immunotherapy: from single candidates to biomarker networks. J Neurol 2008, 255(Suppl 6):48-57.

22. Goertsches R, Zettl U, Hecker M: Sieving treatment biomarkers from blood gene-expression profiles: a pharmacogenomic update on two types of multiple sclerosis therapy. Pharmacogenomics 2011, 12:423-432.

23. Gilli F, Marnetto F, Caldano M, Sala A, Malucchi S, Di Sapio A, Capobianco M, Bertolotto A: Biological responsiveness to first injections of interferon-beta in patients with multiple sclerosis. J Neuroimmunol 2005, 158:195-203.

24. Gilli F, Marnetto F, Caldano M, Sala A, Malucchi S, Capobianco M, Bertolotto A: Biological markers of interferon-beta therapy: comparison among interferon-stimulated genes MxA, TRAIL and XAF-1. Mult Scler 2006, 12:47-57.

25. Pachner A, Narayan K, Price N, Hurd M, Dail D: MxA gene expression analysis as an interferon-beta bioactivity measurement in patients with multiple sclerosis and the identification of antibody-mediated decreased bioactivity. Mol Diagn 2003, 7:17-25.

26. van Baarsen LG, van der Pouw Kraan TC, Kragt JJ, Baggen JM, Rustenburg F, Hooper T, Meilof JF, Fero MJ, Dijkstra CD, Polman CH, Verweij CL: A subtype of multiple sclerosis defined by an activated immune defense program. Genes Immun 2006, 7:522-531.

27. van Baarsen LG, Vosslamber S, Tijssen M, Baggen JM, van der Voort LF, Killestein J, van der Pouw Kraan TC, Polman CH, Verweij CL:

Pharmacogenomics of interferon-beta therapy in multiple sclerosis: baseline IFN signature determines pharmacological differences between patients. PLoS One 2008, 3:e1927.

28. Comabella M, Lunemann JD, Rio J, Sanchez A, Lopez C, Julia E, Fernandez M, Nonell L, Camina-Tato M, Deisenhammer F, Caballero E, Tortola MT, Prinz M, Montalban X, Martin R: A type I interferon signature in monocytes is associated with poor response to interferon-beta in multiple sclerosis. Brain 2009, 132:3353-3365.

29. Bustamante MF, Fissolo N, Rio J, Espejo C, Costa C, Mansilla MJ, Lizasoain I, Angeles Moro M, Carmen Edo M, Montalban X, Comabella M: Implication of the Toll-like receptor 4 pathway in the response to interferon-beta in multiple sclerosis. Ann Neurol 2011, 70:634-645.

30. Axtell RC, de Jong BA, Boniface $K$, van der Voort LF, Bhat R, De Sarno P, Naves R, Han M, Zhong F, Castellanos JG, Mair R, Christakos A, Kolkowitz I, Katz L, Killestein J, Polman CH, de Waal Malefyt R, Steinman L, Raman C: T helper type 1 and 17 cells determine efficacy of interferon-beta in multiple sclerosis and experimental encephalomyelitis. Nat Med 2010, 16:406-412.

31. Feng X, Petraglia AL, Chen M, Byskosh PV, Boos MD, Reder AT: Low expression of interferon-stimulated genes in active multiple sclerosis is linked to subnormal phosphorylation of STAT1. J Neuroimmunol 2002, 129:205-215.

32. van der Voort LF, Vennegoor A, Visser A, Knol DL, Uitdehaag BM, Barkhof F, Oudejans CB, Polman CH, Killestein J: Spontaneous MxA mRNA level predicts relapses in patients with recently diagnosed MS. Neurology 2010, 75:1228-1233. 
33. Hesse D, Krakauer M, Lund $H$, Sondergaard HB, Limborg SJ, Sorensen PS, Sellebjerg F: Disease protection and interleukin-10 induction by endogenous interferon-beta in multiple sclerosis? Eur J Neurol 2010, 18:266-272.

34. Blasius AL, Beutler B: Intracellular toll-like receptors. Immunity 2010, 32:305-315.

35. Bonjardim CA, Ferreira PC, Kroon EG: Interferons: signaling, antiviral and viral evasion. Immunol Lett 2009, 122:1-11.

36. Boo KH, Yang JS: Intrinsic cellular defenses against virus infection by antiviral type I interferon. Yonsei Med J 2010, 51:9-17.

37. Borden EC, Sen GC, Uze G, Silverman RH, Ransohoff RM, Foster GR, Stark GR: Interferons at age 50: past, current and future impact on biomedicine. Nat Rev Drug Discov 2007, 6:975-990.

38. Fensterl V, Sen GC: Interferons and viral infections. Biofactors 2009, 35:14-20.

39. Hall JC, Rosen A: Type I interferons: crucial participants in disease amplification in autoimmunity. Nat Rev Rheumatol 2010, 6:40-49.

40. Haller O, Kochs G, Weber F: Interferon, Mx, and viral countermeasures. Cytokine Growth Factor Rev 2007, 18:425-433.

41. Koyama S, Ishii KJ, Coban C, Akira S: Innate immune response to viral infection. Cytokine 2008, 43:336-341.

42. Sadler AJ, Williams BR: Interferon-inducible antiviral effectors. Nat Rev Immunol 2008, 8:559-568.

43. Takaoka A, Yanai H: Interferon signalling network in innate defence. Cell Microbiol 2006, 8:907-922.

44. Zhang SY, Jouanguy E, Sancho-Shimizu V, von Bernuth H, Yang K, Abel L, Picard C, Puel A, Casanova JL: Human Toll-like receptor-dependent induction of interferons in protective immunity to viruses. Immunol Rev 2007, 220:225-236.

45. McDonald WI, Compston A, Edan G, Goodkin D, Hartung HP, Lublin FD, McFarland HF, Paty DW, Polman CH, Reingold SC, Sandberg-Wollheim M, Sibley W, Thompson A, van den Noort S, Weinshenker BY, Wolinsky JS: Recommended diagnostic criteria for multiple sclerosis: guidelines from the International Panel on the diagnosis of multiple sclerosis. Ann Neurol 2001, 50:121-127.

46. Ferrari F, Bortoluzzi S, Coppe A, Sirota A, Safran M, Shmoish M, Ferrari S, Lancet D, Danieli GA, Bicciato S: Novel definition files for human GeneChips based on GeneAnnot: [http://www.xlab.unimo.it/GA_CDF/].

47. Ferrari F, Bortoluzzi S, Coppe A, Sirota A, Safran M, Shmoish M, Ferrari S, Lancet D, Danieli GA, Bicciato S: Novel definition files for human GeneChips based on GeneAnnot. BMC Bioinforma 2007, 8:446.

48. Hecker M, Goertsches RH, Fatum C, Koczan D, Thiesen HJ, Guthke R, Zett UK: Network analysis of transcriptional regulation in response to intramuscular interferon-beta-1a multiple sclerosis treatment. Pharmacogenomics J 2012, 12:134-146.

49. Goertsches RH, Hecker M, Koczan D, Serrano-Fernandez P, Moeller S, Thiesen HJ, Zettl UK: Long-term genome-wide blood RNA expression profiles yield novel molecular response candidates for IFN-beta-1b treatment in relapsing remitting MS. Pharmacogenomics 2010, 11:147-161.

50. Karlovich C, Duchateau-Nguyen G, Johnson A, McLoughlin P, Navarro M, Fleurbaey C, Steiner L, Tessier M, Nguyen T, Wilhelm-Seiler M, Caulfield JP: A longitudinal study of gene expression in healthy individuals. BMC Med Genet 2009, 2:33.

51. Panne D, Maniatis T, Harrison SC: An atomic model of the interferon-beta enhanceosome. Cell 2007, 129:1111-1123.

52. van Boxel-Dezaire AH, Zula JA, Xu Y, Ransohoff RM, Jacobberger JW, Stark GR: Major differences in the responses of primary human leukocyte subsets to IFN-beta. J Immunol 2010, 185:5888-5899.

53. Sato M, Hata N, Asagiri M, Nakaya T, Taniguchi T, Tanaka N: Positive feedback regulation of type I IFN genes by the IFN-inducible transcription factor IRF-7. FEBS Lett 1998, 441:106-110.

54. Thamilarasan M, Koczan D, Hecker M, Paap B, Zettl UK: MicroRNAs in multiple sclerosis and experimental autoimmune encephalomyelitis. Autoimmun Rev 2012, 11:174-179.

55. Witwer KW, Sisk JM, Gama L, Clements JE: MicroRNA regulation of IFN-beta protein expression: rapid and sensitive modulation of the innate immune response. J Immunol 2010, 184:2369-2376.

56. Sellebjerg F, Krakauer M, Hesse D, Ryder LP, Alsing I, Jensen PE, Koch-Henriksen N, Svejgaard A, Sorensen PS: Identification of new sensitive biomarkers for the in vivo response to interferon-beta treatment in multiple sclerosis using DNA-array evaluation. Eur J Neurol 2009, 16:1291-1298.
57. Dupont SA, Goelz S, Goyal J, Green M: Mechanisms for regulation of cellular responsiveness to human IFN-beta1a. J Interferon Cytokine Res 2002, 22:491-501.

58. Gilli F, Valentino P, Caldano M, Granieri L, Capobianco M, Malucchi S, Sala A, Marnetto F, Bertolotto A: Expression and regulation of IFNalpha/beta receptor in IFNbeta-treated patients with multiple sclerosis. Neurology 2008, 71:1940-1947.

59. Larange A, Antonios D, Pallardy M, Kerdine-Romer S: TLR7 and TLR8 agonists trigger different signaling pathways for human dendritic cell maturation. J Leukoc Biol 2009, 85:673-683.

60. Zhang X, Markovic-Plese S: Interferon beta inhibits the Th17 cell-mediated autoimmune response in patients with relapsing-remitting multiple sclerosis. Clin Neurol Neurosurg 2010, 112:641-645.

61. Jadidi-Niaragh F, Mirshafiey A: Th17 cell, the new player of neuroinflammatory process in multiple sclerosis. Scand J Immunol 2011, 74:1-13.

62. Vandenbroeck K, Alvarez J, Swaminathan B, Alloza I, Matesanz F, Urcelay E, Comabella M, Alcina A, Fedetz M, Ortiz MA, Izquierdo G, Fernandez O, Rodriguez-Ezpeleta N, Matute C, Caillier S, Arroyo R, Montalban X, Oksenberg JR, Antigüedad A, Aransay A: A cytokine gene screen uncovers SOCS1 as genetic risk factor for multiple sclerosis. Genes Immun 2012, 13:21-28.

doi:10.1186/1742-2094-9-140

Cite this article as: Hundeshagen et al: Elevated type I interferon-like activity in a subset of multiple sclerosis patients: molecular basis and clinical relevance. Journal of Neuroinflammation 2012 9:140.

\section{Submit your next manuscript to BioMed Central and take full advantage of:}

- Convenient online submission

- Thorough peer review

- No space constraints or color figure charges

- Immediate publication on acceptance

- Inclusion in PubMed, CAS, Scopus and Google Scholar

- Research which is freely available for redistribution 